\title{
Iron-induced transferrin receptor-1 mRNA destabilization: A response to "Neither miR-7-5p nor miR-141-3p is a major mediator of iron-responsive transferrin receptor-1 mRNA degradation"
}

\author{
MASAKI MIYAZAWA, ${ }^{1}$ ALEXANDER R. BOGDAN, ${ }^{2}$ KAZUNORI HASHIMOTO, ${ }^{3}$ and YOSHIAKI TSUJI ${ }^{4}$ \\ ${ }^{1}$ Department of Health Management, Tokai University, Hiratsuka, Kanagawa, 259-1292 Japan \\ ${ }^{2}$ Minnesota Department of Health, St. Paul, Minnesota 55164, USA \\ ${ }^{3}$ Department of Occupational and Environmental Health, Nagoya University Graduate School of Medicine, Showa-ku, Nagoya, \\ Aichi 466-8550, Japan \\ ${ }^{4}$ Department of Biological Sciences, Toxicology Program, North Carolina State University, Raleigh, North Carolina 27695, USA
}

\begin{abstract}
We read with great interest the Divergent Views article by Connell and colleagues disputing our recent publication describing a role for two microRNAs in the iron-mediated regulation of transferrin receptor 1 (TfR1) mRNA stability. Our publication sought to shed light on a long-standing question in the field of cellular iron metabolism, and we welcome commentary and critique. However, there are several critical issues contained in the article by Connell and colleagues that require further consideration. We appreciate the opportunity to reply here.
\end{abstract}

TfR1 mRNA stability is inversely correlated with intracellular iron concentration; that is, as intracellular iron increases, TfR1 mRNA stability decreases. Specifically, in high iron conditions, RNA-stabilizing proteins called iron regulatory proteins (IRPs) dissociate from five stem-loops called iron response elements (IREs) within the TfR1 mRNA 3'-untranslated region ( $3^{\prime}$-UTR). In their article, Connell and colleagues (Corral et al. 2019) tested TfR1 mRNA stability through the use of luciferase reporter assays by repeating experiments from their previous publication (Rupani and Connell 2016) as well as testing the miR-7-5p and miR$141-3 p$ target sites we recently characterized within the context of their experimental system.

In the article, Connell and colleagues showed that introducing mutations in the miR target sites in their TfR1 reporter system failed to increase luciferase expression while their mutants in the region between IRE-B and $-C$ increased luciferase, claiming that TfR1 mRNA was not stabilized even when the miR target site was destroyed. However, it is plausible that this discrepancy is primarily due to their luciferase assay set-up that used only an iron-replete condition (+iron) without taking an untreated (i.e., no additional iron [-iron]) control. They compared

Corresponding authors: m.miyazawa@tsc.u-tokai.ac.jp, ytsuji@ ncsu.edu

Article is online at http://www.rnajournal.org/cgi/doi/10.1261/rna. 073270.119 luciferase expression only between wild-type and mutant reporters in the iron-replete condition rather than comparing \pm iron within the same reporter construct like we did (Miyazawa et al. 2018). This assay condition and approach were also used in their previous work to minimize potential complications from IRP binding and protection (Rupani and Connell 2016). Directly comparing luciferase activities from construct-to-construct or even batch-to-batch can be extremely difficult in a transient transfection setting; variations in the plasmid sequence or differences in plasmid purity can cause wildly different baseline luciferase activities. For this reason and more importantly to measure luciferase reporter expression under the condition of iron-induced TfR1 mRNA degradation via the IRP-IRE system, we performed comparisons between untreated and iron-replete conditions within a single construct rather than comparing across different plasmid sequences (Fig. 3 in Miyazawa et al. 2018). In addition, by forcing IRP dissociation through the addition of iron to push TfR1 mRNA into an unstable form, without also comparing to an untreated control, it is unknown what effect their mutations are having on IRP

(C) 2019 Miyazawa et al. This article is distributed exclusively by the RNA Society for the first 12 months after the full-issue publication date (see http://rnajournal.csh/p.org/site/misc/terms.xhtml). After 12 months, it is available under a Creative Commons License (Attribution-NonCommercial 4.0 International), as described at http:// creativecommons.org/licenses/by-nc/4.0/. 
binding ability and TfR1 mRNA stability under physiological conditions. Moreover, Connell et al. made several new IRE mutant reporters that attempted to destroy the miR target sites but never tested the impact of these mutations on binding to IRPs. Indeed, some of their mutants (e.g., IRE-C \#3) not only destroyed the miR-7-5p target site but also changed almost the entire IRE-C sequences including the conserved loop sequence that is essential for interaction with IRPs (Sierzputowska-Gracz et al. 1995; Laing and Hall 1996; Menotti et al. 1998; Kühn 2015). Additionally, Connell et al. did not observe the effect of miR-7-5p transfection on their reporters and TfR1 mRNA. This experiment was also performed only in an iron-replete condition without taking an untreated (-iron) control. Providing more specific issues below that underscore the potential clues for the discrepancy, we think that their atypical reporter assays and the lack of an untreated (-iron) control may primarily characterize stabilization mutations of the TfR1 mRNA in an IRP-IRE-independent manner.

1. Connell et al. used a "minimized" TfR1 3'-UTR luciferase reporter (Fig. 1A, a 245-base region containing only IRE$B,-C$, and $-D$ stem-loops and an approximately 270base truncation between the IRE- $B$ and IRE- $C$ region, a repetition of previously published Fig. 3A in Rupani and Connell 2016). In Figure 1D, they tested the $\Delta 149-177$ mutant lacking the entire miR-7-5p binding site and observed no significant impact on luciferase expression compared to wild-type in SW480 cells we used. However, this mutation deletes the entire IRE-C stem-loop but surprisingly it behaved like wild-type in their reporter assay in Fig. 1D. The fact that the deletion of the IRE-C from what they call "minimized" TfR1 3'UTR had no impact clearly indicates that their stability measure of TfR1 mRNA is independent of IRE-C. This is inconsistent with the reports published previously (Casey et al. 1988; Müllner and Kühn 1988; Erlitzki et al. 2002). It should also be noted that this group's RNA-CLIP data to pull down IRP1 binding sequences demonstrated that the predominant crosslinked sites in TfR mRNA were IRE-C (Rupani and Connell 2016). Therefore, their measure of TfR1 mRNA is apparently outside the context of the IRP-IRE system. If no effect by deletion of IRE-C would be explained due to their iron-replete condition used throughout their assays, it suggests that their approaches are primarily to map regulatory regions of TfR1 mRNA stability in an iron-independent manner.

2. In Figure 2D, Connell et al. used another reporter termed "extended" $3^{\prime}$-UTR that contains all five IREs of TfR1 and tested four mutants (IRE-C mut \#3, IRE-E mut \#1, IRE-E deletion $\triangle \mathrm{IRE}-\mathrm{E}$, and $\mathrm{C} 78 \mathrm{G}$ ) to repeat the luciferase assays in SW480 cells. We noticed that the IRE-C mut \#3 as well as IRE-E mut \#1 in Figure 2D may still keep the stem-loop structure but their IRE-C and IRE-E sequences were totally destroyed including the conserved loop sequence CAGUG/A critically involved in binding to IRPs (Sierzputowska-Gracz et al. 1995; Laing and Hall 1996; Menotti et al. 1998; Kühn 2015). As IRP binding ability to such heavily mutated sequences was not tested, it is unknown if and to what degree IRP binding to these constructs was altered. Given that the conserved IRE loop sequence is essential for interaction with IRPs (Sierzputowska-Gracz et al. 1995; Henderson et al. 1996; Menotti et al. 1998; Kühn 2015) and our recent ferritin IRE study (Miyazawa et al. 2019), we doubt about retaining their IRE functions. In other words, all three IRE mutants tested in Figure 2D not only destroyed the miR target sites but potentially also impaired IRE-C and IRE-E function. Despite that, their interpretation of results in Figure 2D was that C78G is a stabilizing mutation, whereas none of the IRE-C and IRE-E mutants that disrupted miR-7-5p and miR-141-3p target sites have any stabilizing effect. Under the influence of loss of the IRE function in their mutation assays, the outcome is more complicated, difficult to be interpreted, and does not support their conclusion. This issue is also seen in Figure 3 when they used an optimized $3^{\prime}$ UTR reporter. An additional note here is that Figure 3A by Connell et al. is not from this work but a repetition of their previously published Figure 3B (Rupani and Connell 2016).

3. Another important issue that explains their claim of no impact of mutation/deletion of the miR target sites in Figure 2D is the lack of control in their luciferase assays; namely, they only used an iron-replete condition (+iron) without comparing to no additional iron (-iron) within the same reporter construct. The major issue is that their assay performed only in the iron-replete condition was not aimed to identify stabilization mutations within the context of the IRP-IRE system. For instance, if the ratio of luciferase expression between \pm iron in wild-type IRE reporter is $50 \%$ (50\% decrease in luciferase expression after adding iron), what is this ratio in their stabilization mutants, such as C78G in Figure 2D? If the C78G mutant also shows $50 \%$ decrease in (+iron) compared to (-iron) conditions, it suggests that the C78G mutation, behaving like wild-type IRE, does not affect TfR1 mRNA stability regulated by the IRP-IRE system. In contrast, if the C78G mutant shows only $10 \%$ decrease in luciferase expression after adding iron while 50\% decrease in wild-type IRE reporter, it suggests that this mutation blocks iron-induced TfR1 mRNA degradation under the IRP-IRE system. Therefore, the set-up of luciferase assays is fundamentally different between ours and Connell et al.

As mentioned above, it is very likely that their IRE-C and IRE-E mutants in Figure 2D lost not only our miR 
A

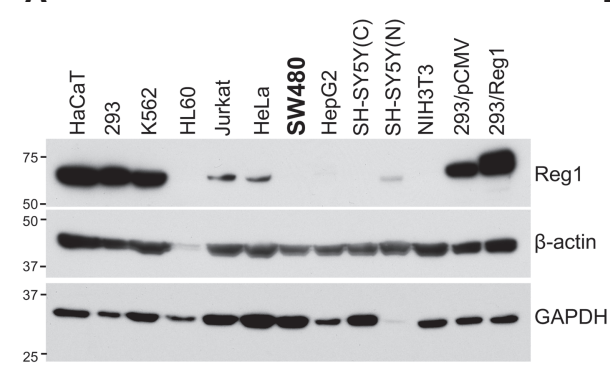

B

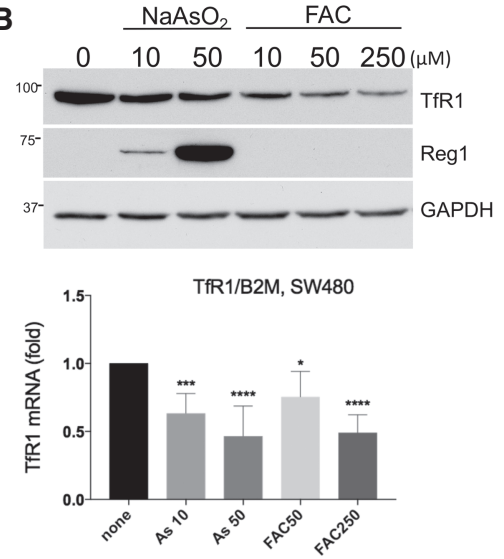

FIGURE 1. Reg1 expression in various cell lines and the effects of Reg1 on endogenous TfR1 expression. (A) Thirty micrograms of whole cell lysates isolated from nine cell lines and cytoplasmic $(\mathrm{C})$ and nuclear $(\mathrm{N})$ fractions from SH-SY5Y cells were loaded on SDS-PAGE and subjected to western blotting with anti-ZC3H12A (Reg1) antibody (GeneTex, GTX110807), anti- $\beta$ actin antibody (SIGMA, A5441), and anti-GAPDH (Chemicon, MAB374). (B) SW480 cells were treated with 10,50 $\mu \mathrm{M} \mathrm{NaAsO}$, or 10, 50, $250 \mu \mathrm{M} \mathrm{FAC} \mathrm{(ferric} \mathrm{ammonium} \mathrm{citrate)} \mathrm{for} 18 \mathrm{~h}$ and harvested for preparation of whole cell lysates and total RNA. (Top) TfR1 protein expression was detected by western blotting with anti-TfR1 antibody (Abcam ab84036), followed by Reg1 and GAPDH antibodies. (Bottom) Total RNA was subjected to CDNA synthesis and qPCR according to Miyazawa et al. (2018). TfR1 Cq was normalized with B2M Cq ( $\beta 2$-microglobulin), and eight qPCR results from three experiments were statistically analyzed by one-way ANOVA with Prizm 7. $P$-values compared to none; $\left(^{*}\right) P=0.0124,\left({ }^{* *}\right) P=0.0002,\left(^{* * * *}\right) P=$ 0.0001 .

target site but also the IRE function due to the massive sequence changes including the CAGUG loop sequence. We agree with their interpretation of $\mathrm{C} 78 \mathrm{G}$ as a stabilization mutation and used as a positive control in this assay but we disagree with the interpretation of the results in their IRE-C and IRE-E mutants in Figure $2 \mathrm{D}$ because their mutants cannot assess the specific impact on the miR target sites. Without knowing the basal expression in (-iron) condition and more targeted mutations throughout their experiments, we have no way to fairly evaluate the effects of iron on their miR-targeted reporter constructs.

4. Connell et al. also claimed that overexpression of miR7-5p did not impact on their luciferase reporter or endogenous TfR1 mRNA in Fig. 4. We think that their reliance on the iron-replete condition placed their assays more difficult to get clear differences between one construct and another or between miR-Con and miR-7-5p. The iron-replete condition means that they already activated iron-induced degradation mechanisms of TfR1 mRNA in all reporter-transfected cells. We showed that the transfection of miR-7-5p in the iron-replete condition ( $250 \mu \mathrm{M}$ FAC) could not push further degradation of TfR1 mRNA. As shown in our Figure 2B by Miyazawa et al. (2018), the magnitude of difference in TfR1 mRNA between iron-treated miR-Con and iron-treated miR-7$5 p$, while statistically significant, was still smaller than the difference between miR-Con and miR-7-5p in the (-iron) condition (Fig. 2B by Miyazawa et al. 2018); intuitively, this makes sense as it would be expected that the expression change from stable TfR1 mRNA (without additional iron) to unstable TfR1 mRNA (iron-replete) would be greater than trying to further destabilize already unstable TfR1 mRNA (the addition of miR7-5p/miR-141-3p to iron-replete conditions). The same reason can be applied to no impact on endogenous TfR1 mRNA by their miR-7$5 p$ mimic transfection because they treated all transfected cells with $65 \mu \mathrm{g} / \mathrm{mL}(245 \mu \mathrm{M}) \mathrm{FAC}$ for 5 $\mathrm{h}$ prior to harvesting RNA. This $5 \mathrm{~h}$ iron-replete condition was enough to induce TfR1 mRNA degradation (Koeller et al. 1991; Kühn 2015; Miyazawa et al. 2018) but had no effect on EGF-R mRNA in SW480 cells (Fig. 2B by Miyazawa et al. 2018). Therefore, the impact of transfected miR-7-5p under an already activated iron-induced degradation system on the stability of TfR1 mRNA (but not EGF-R mRNA) is expected to be much smaller, or potentially unmeasurable, under iron-replete conditions.

5. This work by Connell and colleagues appears to be mainly an effort to confirm their idea of the loops I, III, and V localized in the "minimized" truncated TfR1 region spanning from IRE-B to IRE-D with little new experimental evidence for what the TfR1 mRNA degradation pathway is. In our previous publication, we already took a significant part of the discussion for mechanisms other than microRNAs which might play a major role in ironmediated TfR1 mRNA degradation (Miyazawa et al. 2018). Our discussion of alternative mechanisms in our previous work was based on the results that we did not observe clear blocking effects by antagomiR-7 and -141 on iron-induced TfR1 mRNA degradation, particularly in some mouse cell lines such as NIH3T3 cells (Fig. 4 by Miyazawa et al. 2018) despite the fact that the TfR1 mRNA stability regulation via the IRPIRE system is highly conserved among vertebrate species including mouse (Theil 1994; Piccinelli and Samuelsson 2007). We also did not observe significant effects of miR-141-3p in K562 cells (Fig. 2C by Miyazawa et al. 2018). We acknowledged that other mechanisms may dominate over microRNAs and discussed several possibilities at length, including 
Regnase-1 (Reg1) as mentioned in the article by Connell et al. It is a plausible idea and was indeed demonstrated (Yoshinaga et al. 2017). We also preliminary tested Reg1 whether it might be a major endonuclease within the context of the iron-regulated IRP-IRE system and briefly discussed it (Miyazawa et al. 2018). Reg1 is an endonuclease for TfR1 mRNA destabilization (Yoshinaga et al. 2017); however, we think that it needs further assessment whether Reg1 works within the context of the IRP-IRE system for the following reasons;

a. It was demonstrated in TfR1 3'-UTR luciferase reporter assays that Reg1 exhibited dose (input of plasmid DNA) dependent decrease in luciferase expression enhanced by cotransfected IRPs (Fig. 21 in Yoshinaga et al. 2017). It may be due to steric competition between Reg1 and IRPs as discussed in Yoshinaga et al. (2017), or Reg1 and IRPs may work independently. They also found that iron deficiency induced Reg1 expression via HIF2 $\alpha$ (Yoshinaga et al. 2017). As iron deficiency drives TfR1 mRNA stabilization rather than degradation via the IRP-IRE system, the role of Reg1 in TfR1 mRNA destabilization may not be necessarily associated with the IRP-IRE system. In this regard, the possibility of competition between Reg1 and IRPs was proposed but not further tested (Yoshinaga et al. 2017).

b. As noted in our publication (Miyazawa et al. 2018) as well as Corral et al. (2019), Yoshinaga et al. (2017) successfully identified a Reg1 target site as a conserved small stem-loop in close proximity to TfR1 IRE. We noticed that this Reg1 target is identical to the loop III reported by this group (Rupani and Connell 2016). The C78G mutation in their luciferase reporter is in the loop III, assuming that the C78G mutation may block the Reg1 attack. Therefore, this C78G mutant seems to be a Reg1-resistant TfR1 stability mutant probably regardless of the cellular iron status.

c. We preliminarily tested Reg1 expression by western blotting in various cell lines including SW480 we primarily used in our previous work (Miyazawa et al. 2018). We found that Reg1 expression was extremely low to undetectable in SW480 and in several other cell lines we tested under regular culture conditions (Fig. 1A). We found that sodium arsenite $\left(\mathrm{NaAsO}_{2}\right)$ but not FAC strongly induced Reg1 in SW480 cells (Fig. 1B). Sodium arsenite decreased TfR1 expression (Fig. 1B) either directly by Reg1 and/or indirectly as a result of its antiproliferative effect or cytotoxicity. FAC treatment did not induce Reg1 expression (still undetectable) but induced TfR1 downregulation (Fig. 1B). Therefore, it is unlikely that Reg1 plays an endonuclease role in TfR1 mRNA degradation, at least in SW480 cells, within the context of the IRP-IRE system. d. The results of luciferase reporter assays in Figure 1D and Figure 2D by Connell et al. showed increased luciferase expression by the C78G mutation in SW480 cells. Given that Reg1 expression in SW480 is undetectable even in the iron-replete condition (Fig. 1) and the C78G mutation is in the Reg1 targeted stem-loop (Yoshinaga et al. 2017), overall it is extremely difficult to reconcile most of the results presented by Connell and colleagues.

To conclude, we appreciate the evaluation by Connell and colleagues of the miR-7-5p and miR-141-3p sites located within the TfR1 3'UTR we previously characterized. However, significant questions remain about the experimental system used: (i) their mutants are not specific to destroy only the miR target sites, and (ii) most significantly, using iron-replete conditions without an untreated control ignores the role of IRPs and investigates TfR1 mRNA only in an already unstable condition, making interpretation of the results extremely difficult and drawing definitive conclusions unfeasible. Additionally, while Reg1 was previously identified as an endonuclease that targets TfR1 within the IRE region as discussed in our manuscript (Miyazawa et al. 2018), there are still outstanding questions about whether Reg1 is indeed responsible for iron-mediated TfR1 mRNA degradation or if it is acting independently of the IRE-IRP system.

\section{REFERENCES}

Casey JL, Hentze MW, Koeller DM, Caughman SW, Rouault TA, Klausner RD, Harford JB. 1988. Iron-responsive elements: regulatory RNA sequences that control mRNA levels and translation. Science 240: 924-928. doi:10.1126/science.2452485

Corral VM, Schultz ER, Connell GJ. 2019. Neither miR-7-5p nor miR$141-3 p$ is a major mediator of iron-responsive transferrin receptor-1 mRNA degradation. RNA 25: 1407-1415 (this issue). doi: 10.1261/rna.072371.119

Erlitzki R, Long JC, Theil EC. 2002. Multiple, conserved iron-responsive elements in the $3^{\prime}$-untranslated region of transferrin receptor mRNA enhance binding of iron regulatory protein 2. J Biol Chem 277: 42579-42587. doi:10.1074/jbc.M207918200

Henderson BR, Menotti E, Kühn LC. 1996. Iron regulatory proteins 1 and 2 bind distinct sets of RNA target sequences. J Biol Chem 271: 4900-4908. doi:10.1074/jbc.271.9.4900

Koeller DM, Horowitz JA, Casey JL, Klausner RD, Harford JB. 1991. Translation and the stability of mRNAs encoding the transferrin receptor and c-fos. Proc Natl Acad Sci 88: 7778-7782. doi:10.1073/ pnas.88.17.7778

Kühn LC. 2015. Iron regulatory proteins and their role in controlling iron metabolism. Metallomics 7: 232-243. doi:10.1039/ C4MT00164H

Laing LG, Hall KB. 1996. A model of the iron responsive element RNA hairpin loop structure determined from NMR and thermodynamic data. Biochemistry 35: 13586-13596. doi:10.1021/ bi961310q

Menotti E, Henderson BR, Kühn LC. 1998. Translational regulation of mRNAs with distinct IRE sequences by iron regulatory 


\section{Miyazawa et al.}

proteins 1 and 2. J Biol Chem 273: 1821-1824. doi:10.1074/jbc .273.3.1821

Miyazawa M, Bogdan AR, Hashimoto K, Tsuji Y. 2018. Regulation of transferrin receptor-1 mRNA by the interplay between IRE-binding proteins and miR-7/miR-141 in the 3'-IRE stem-loops. RNA 24: 468-479. doi:10.1261/rna.063941.117

Miyazawa M, Bogdan AR, Tsuji Y. 2019. Perturbation of iron metabolism by cisplatin through inhibition of iron regulatory protein 2. Cell Chem Biol 26: 85-97.e4. doi:10.1016/j.chembiol.2018.10 .009

Müllner EW, Kühn LC. 1988. A stem-loop in the $3^{\prime}$ untranslated region mediates iron-dependent regulation of transferrin receptor mRNA stability in the cytoplasm. Cell 53: 815-825. doi:10.1016/00928674(88)90098-0

Piccinelli P, Samuelsson T. 2007. Evolution of the iron-responsive element. RNA 13: 952-966. doi:10.1261/rna.464807
Rupani DN, Connell GJ. 2016. Transferrin receptor mRNA interactions contributing to iron homeostasis. RNA 22: 1271-1282. doi:10 1261/rna.056184.116

Sierzputowska-Gracz H, McKenzie RA, Theil EC. 1995. The importance of a single $G$ in the hairpin loop of the iron responsive element (IRE) in ferritin mRNA for structure: an NMR spectroscopy study. Nucleic Acids Res 23: 146-153. doi:10.1093/nar/ 23.1.146

Theil EC. 1994. Iron regulatory elements (IREs): a family of mRNA noncoding sequences. Biochem J 304(Pt 1): 1-11. doi:10.1042/ bj3040001

Yoshinaga M, Nakatsuka Y, Vandenbon A, Ori D, Uehata T, Tsujimura T, Suzuki Y, Mino T, Takeuchi O. 2017. Regnase-1 maintains iron homeostasis via the degradation of transferrin receptor 1 and prolyl-hydroxylase-domain-containing protein 3 mRNAs. Cell Rep 19: 1614-1630. doi:10.1016/j.celrep.2017.05.009 

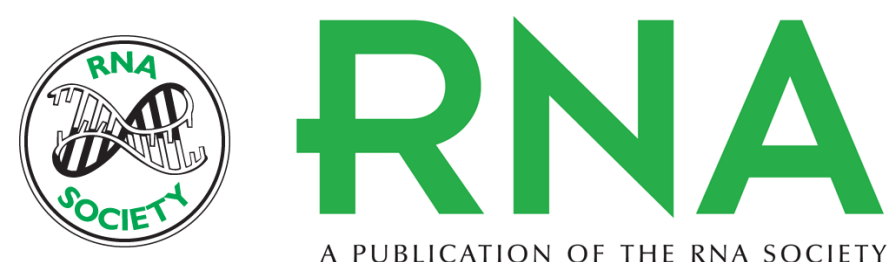

A PUBLICATION OF THE RNA SOCIETY

\section{Iron-induced transferrin receptor-1 mRNA destabilization: A response to "Neither miR-7-5p nor miR-141-3p is a major mediator of iron-responsive transferrin receptor-1 mRNA degradation"}

Masaki Miyazawa, Alexander R. Bogdan, Kazunori Hashimoto, et al.

RNA 2019 25: 1416-1420 originally published online September 11, 2019

Access the most recent version at doi:10.1261/rna.073270.119

References This article cites 16 articles, 10 of which can be accessed free at:

http://rnajournal.cshlp.org/content/25/11/1416.full.html\#ref-list-1

Articles cited in:

http://rnajournal.cshlp.org/content/25/11/1416.full.html\#related-urls

Creative This article is distributed exclusively by the RNA Society for the first 12 months after the

Commons full-issue publication date (see http://rnajournal.cshlp.org/site/misc/terms.xhtml). After 12

License months, it is available under a Creative Commons License (Attribution-NonCommercial 4.0 International), as described at http://creativecommons.org/licenses/by-nc/4.0/.

Email Alerting Receive free email alerts when new articles cite this article - sign up in the box at the Service top right corner of the article or click here.

To subscribe to RNA go to:

http://rnajournal.cshlp.org/subscriptions 